\title{
Experiencias de la instauración de normas relativas al contenido de plomo en aire y el agua, en los Estados Unidos de América
}

\author{
J Michael D avis, Ph D, (1) Lester D Grant, Ph D.(1)
}

\begin{abstract}
Davis JM, Grant LD. Experiencias de la instauración de normas relativas al contenido de plomo en aire y el agua, en los Estados Unidos de América.

Salud Publica Mex 2003;45 supl 2:S237-S243. El texto completo en inglés de este artículo está disponible en: http://www.insp.mx/salud/index.html

\section{Resumen}

En los Estados U nidos de A mérica se ha logrado una disminución de la exposición a plomo de la población en general, gracias al establecimiento de diversas normas. En este artículo se destaca parte de los conocimientos adquiridos a través de la experiencia obtenida en este país en materia de plomo, en particular respecto al aire y al agua. Uno de los aspectos fundamentales es la posibilidad de contar con una base científica sólidamente establecida y claramente entendida, a partir de la cual se tomarán las medidas correspondientes. Los efectos secundarios de ciertas normas concernientes al aire ponen de manifiesto de qué manera los esfuerzos orientados a solucio nar un problema pueden aportar beneficios en otras áreas, si bien pueden surgir consecuencias no deseadas, debido a la falta de previsión y de evaluación. Asimismo, en este documento se discuten las diferencias entre los enfo ques centralizados y los descentralizados para el manejo de la exposición a plomo. El texto completo en inglés de este artículo está disponible en:http:/ /www.insp.mx/salud/index.html
\end{abstract}

Palabras clave: plomo; contaminación del aire; contaminación del agua; exposición; legislación ambiental; Estados U nidos

\author{
Davis JM, Grant LD. \\ Establishing lead in air \\ and water standards \\ in the United States of America. \\ Salud Publica Mex 2003;45 suppl 2:S237-S243. \\ The English version of this paper \\ is available at: http://www.insp.mx/salud/index.html
}

\begin{abstract}
A bstract
Lead regulations and standards have resulted in a lower exposure to lead in the general population of the United States of America. This paper highlights some of the knowhow developed through lead-containing experiences, particularly regarding lead content in air and water. The availability of a solid and clear scientific knowledge is central to the success of these policies. Collateral effects of some air-related standards show the extent to which problemoriented efforts may be beneficial in other areas, inasmuch as they may have untoward consequences if careful planning and evaluation are not considered. Finally, this paper presents a discussion of the differences between centralized and decentralized approaches to lead exposure control.The English version of this paper is available at:http://www.insp.mx/salud/ index.html
\end{abstract}

Key words: lead; air pollution; water pollution; legislation; environment; U nited States

(1) Centro $\mathrm{N}$ acional de Evaluación Ambiental, 0 ficina de Investigación y Desarrollo, A gencia N orteamericana para la Protección del A mbiente, Estados Unidos de América.

Fecha de recibido: 1 de febrero de 2002 - Fecha de aprobado: 9 de mayo de 2002 Solicitud de sobretiros: Dr.J Michael Davis. Centro N acional de Evaluación Ambiental - RTP (MD-52),Agencia N orteamericana para la Protección del A mbiente. Research Triangle Park, N C 27711, Estados Unidos de América. Correo electrónico: D avis.Jmichael.@ epa.gov 
L a Agencia Norteamericana para la Protección del Ambiente (USEPA, por sus siglas en inglés) ha tomado diversas medidas reguladoras que, de manera directa o indirecta, han tenido un efecto sobre la exposición de la población en general al plomo en el aire o en el agua. Un mayor conocimiento de sus efectos sobre la salud ha permitido sustentar, sobre bases sólidas, el fundamento científico para llevar a cabo dichas acciones. En este trabajo se destacan algunos puntos clave de la experiencia que se ha tenido en los Estados Unidos de América (EUA) con este metal, y se incluyen algunas enseñanzas, producto de estas experiencias, que pueden resultar didácticas para aquellos interesados en desarrollar políticas y estrategias de salud pública en otros países o, eventualmente, para el caso de otros contaminantes ambientales. Este artículo se elaboró a partir de trabajos previos de los autores, ${ }^{1,2}$ que se pueden consultar si se desea obtener información adicional.

\section{Fundamento científico}

Una buena política de salud pública debe basarse en el conocimiento científico de los riesgos asociados con un peligro potencial. Ciertamente, el conocimiento acerca de éstos puede servir como incentivo, tanto al interior de la salud pública comunitaria, como en la sociedad en general, para formular las políticas de salud pública que permitan responder a riesgos identificados. En el caso del plomo, durante las décadas pasadas se ha desarrollado una vasta red de información relativa a sus efectos sobre la salud..$^{3,4}$ En la medida en que ha ido surgiendo más información confiable sobre este tema se han ido identificando, progresivamente, niveles de exposición más y más bajos, como una amenaza para la salud pública. Así, por ejemplo, el nivel de plomo en sangre (NPS) de niños en los cuales se pensaba que la intervención quedaba justificada, en la década de 1960 descendió alrededor de los 60 a los 40 y, después, a los $30 \mu \mathrm{g} / \mathrm{dl}$ en la década de 1970; después, bajó a los $25 \mu \mathrm{g} / \mathrm{dl}$ en la de los ochenta, y a 10 en la de los noventa. ${ }^{5}$ Ya desde 1986 la USEPA había concluido, partiendo de una amplia revisión de los estudios disponibles que incluían los estudios epidemiológicos prospectivos que entonces surgían (emerging prospective epidemiological studies), además de diversos estudios de otro tipo en humanos y animales, que "los niveles de plomo en sangre perinatales de 10 a $15 \mu \mathrm{g} / \mathrm{dl}$ significan, indudablemente, un motivo de preocupación, debido a sus efectos nocivos en el desarrollo pre y postnatal". ${ }^{3}$ Conviene observar que estos niveles no se consideraban como un umbral de toxicidad, puesto que se reconocía la posibilidad de que hubiese efectos nocivos en niveles por debajo de los $10 \mu \mathrm{g} / \mathrm{dl}$. Además, si bien la inquietud que ocasionaba su presencia estaba particularmente bien sustentada en la evidencia de los efectos de la exposición a plomo de bajo nivel en el desarrollo neurológico y conductual en los niños, surgió más evidencia en su favor a partir de los estudios experimentales hechos en animales, así como de un espectro de los efectos en la salud humana que incluía rezago en el crecimiento infantil, incremento de la presión arterial en los varones adultos, daños en la hemosíntesis y en el metabolismo de la vitamina $D$, y alteraciones neuro-electrofisiológicas en los potenciales evocados, en la velocidad de la conducción nerviosa y en la agudeza acústica. ${ }^{3} \mathrm{La}$ conclusión de la USEPA de que un NPS de "10 a $15 \mu \mathrm{g} / \mathrm{dl}$, y probablemente otros más bajos aún" resultaban preocupantes, fue confirmada posteriormente por otros organismos encargados de la salud pública, incluyendo la Agencia para el Registro de la Enfermedad y Substancias Tóxicas (Agency for Toxic Substances and Disease Registry), ${ }^{6,7}$ los Centros para la Detección y el Control de las Enfermedades ${ }^{5}$, y la Organización Mundial de la Salud. ${ }^{8}$

Es importante aclarar que en ese nivel se hace referencia a poblaciones enteras; un individuo en particular puede no necesariamente manifestar signos fácilmente detectables de daño neuroconductual o de otro tipo, con un NPS de $10 \mu \mathrm{g} / \mathrm{dl}$; sin embargo, la tendencia a la baja, estadísticamente significativa, en las mediciones de las funciones cognitivas, así como de otros aspectos neuroconductuales, han quedado bien documentadas en grupos de niños que presentan NPS de alrededor de $10 \mu \mathrm{g} / \mathrm{dl}$. La imposibilidad de extrapolar de lo general a lo particular en ocasiones parecería confundir la percepción del significado que, en materia de salud pública, tienen los efectos del plomo en niveles bajos. Para algunos parecería que la sutileza de una disminución de 2 a 4 puntos en el promedio del coeficiente intelectual de una población infantil fuese muy poco relevante, en comparación con las convulsiones y otros signos propios de la encefalopatía en un niño francamente intoxicado. La ocurrencia de este último fenómeno representa, sin lugar a dudas, un grave problema, tanto para las personas directamente involucradas como para la sociedad en su conjunto. Sin embargo, los efectos menos obvios de la exposición de bajo nivel a plomo también ameritan atención en tanto que se trata de un problema igualmente grave, pues la cifra de sujetos afectados puede llegar a ser considerable.

En la figura 1 se ilustra, tridimensionalmente, un cambio observado en las distribuciones de las calificaciones relativas al coeficiente intelectual como una fun- 


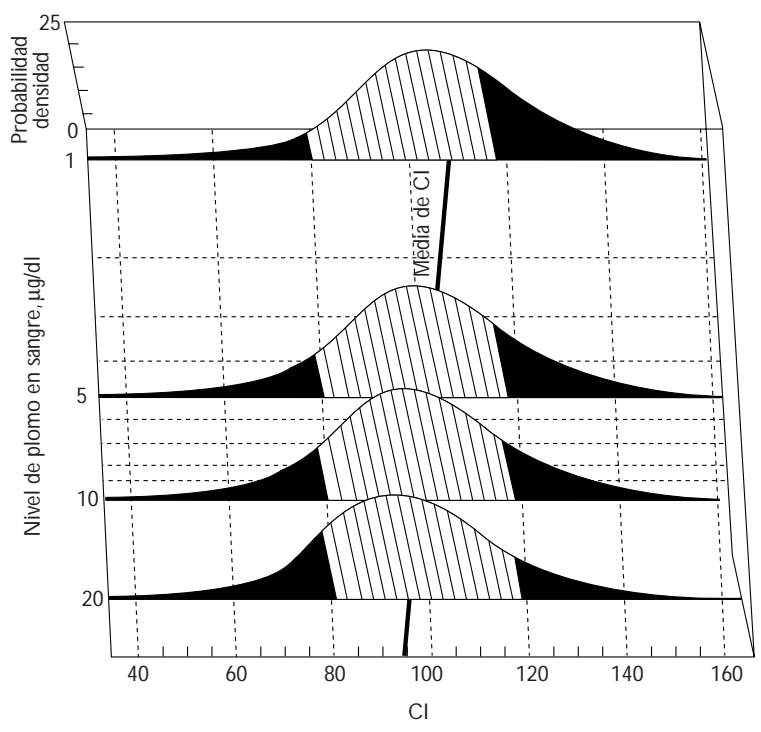

Fuente: Referencia 9

Figura 1. Distribuciones normales de los puntajes DE COEFICIENTE INTELECTUAL CON UNA DESVIACIÓN ESTÁNDAR DE 16, Y MEDIAS QUE VAN DE 108 (EN UN NIVEL PROMEDIO DE PLOMO EN SANGRE DE $1 \mu \mathrm{G} / \mathrm{DL}$ ) A 96 (EN UN NIVEL PROMEDIO DE PLOMO EN SANGRE DE $20 \mu \mathrm{G} / \mathrm{DL}$ )

ción de los cambios en los NPS de las poblaciones. ${ }^{9}$ Cuando estos niveles van de 1 a $20 \mu \mathrm{g} / \mathrm{dl}$, se representan en una escala logarítmica que va de atrás hacia adelante. Los coeficientes de inteligencia (CI) de 40 a 160 se muestran de izquierda a derecha, en tanto que el eje vertical representa la probabilidad de tener distintos puntajes de CI en una población, partiendo del supuesto de una distribución normal, con una desviación estándar de 16. Tal cual se ilustra en dicha figura, el promedio de $\mathrm{CI}$ es de 108 para la población con un nivel de plomo en sangre de $\mu \mathrm{g} / \mathrm{dl}$, comparado con la mediana de 96 cuando los NPS son de $20 \mu \mathrm{g} / \mathrm{dl}$. Las implicaciones de un cambio de este tipo en los niveles promedio de $\mathrm{CI}$ son particularmente conspicuas cuando se examinan las colas de distribución. Cabe observar cómo el área de distribución que representa los CI de 80 o menos se incrementa cuando los NPS son más elevados, o bien cómo decrece la cifra de CI de 120 o más. Si bien es posible que la representación gráfica no logre mostrarlo de manera obvia, la cantidad de puntajes, en al menos una desviación estándar por debajo de la media, aumenta en $50 \%$ por cada dismi- nución de cuatro puntos en el CI promedio. En combinación con los cambios inversos en la cola superior de la distribución, éstos, en el puntaje de CI de la población, implican un tremendo costo potencial para la sociedad, por una parte debido a la necesidad de destinar más recursos para ayudar a los que se ubican en la cola inferior y, por la otra, en términos de los beneficios que se pierden al no tener a más de los "mejores y más brillantes" en la cola superior.

Las implicaciones de estos efectos de la exposición de bajo nivel a plomo pueden ser más tangibles cuando se traducen en costos y beneficios desde el punto de vista monetario. Como lo observara Schwartz: ${ }^{10}$ "En nuestra sociedad, la gente sigue estando expuesta al plomo no porque se piense que la exposición es buena, sino porque reducirla cuesta dinero. Empero, el hecho de mantener la exposición también tiene sus costos". En 1986 la USEPA calculó que, en los EUA, la eliminación del contenido del metal en la gasolina tendría un costo anual de más de 500 millones de dólares. Sin embargo, los beneficios de esta medida, al traducirlos en la reducción de costos y en el aumento de ganancias asociadas con los cambios en el CI representado en la figura 1 , junto con muchos otros beneficios en materia de salud que implica la reducción de la exposición a plomo, rebasaban considerablemente los costos económicos de su eliminación. Un análisis de costo-beneficio llevado a cabo por la EPA en relación con los estándares del agua potable también mostró que el incremento en los costos que implicaba el establecimiento de controles más rígidos con respecto al plomo, no significaba gran cosa al compararlo con los beneficios que se obtenían al disminuir los requerimientos de atención médica, de educación especial y demás ramificaciones de la exposición a este metal.

\section{El plomo en el aire}

Tradicionalmente, las fuentes de emisión a la atmósfera se han clasificado como móviles, por ejemplo, los automóviles y los camiones, y estacionarias, por ejemplo, las fundidoras y las plantas generadoras de energía eléctrica. Las fuentes móviles de emisión del metal comenzaron a declinar en los EUA cuando en 1973 se introdujeron los convertidores catalíticos en los automóviles. La gasolina con plomo corrompía los catalizadores disminuyendo considerablemente su capacidad para eliminar los contaminantes del motor que iban al tubo de escape, como, por ejemplo, el monóxido de carbono y los hidrocarburos. Así entonces, el interés fundamental en abandonar el consumo de esta gasolina fue, inicialmente, no el de reducir la exposición humana al metal, sino más bien proteger los arte- 
factos de control de emisiones necesarios para reducir el contenido de otros contaminantes. Sin embargo, el efecto de la disminución en el consumo de esta gasolina se hizo evidente muy pronto, tanto en los niveles de plomo existentes en la atmósfera (figura 2), como en aquellos en sangre humana (figura 3). ${ }^{11}$ Existen otros países, como Suiza, en donde también se han observado correlaciones similares al cambiar al consumo de gasolina sin el metal. ${ }^{12}$ En la medida en la que se pudo apreciar la efectividad de la reducción en el consumo de gasolina, respecto a los niveles de plomo en el aire y en la sangre, y que los riesgos a la salud debidos a una exposición baja pudieron definirse mejor, ${ }^{13}$ se estableció la necesidad de disminuir aún más los contenidos de plomo en la gasolina en los EUA, al final de la década de los ochenta e inicios de los noventa. ${ }^{13,14}$

Las emisiones del metal a partir de fuentes estacionarias también han disminuido durante las dos últimas décadas. ${ }^{15}$ Parte de esta disminución se debe a la Norma Nacional para la Calidad del Aire en el Ambiente (NAAQS, por sus siglas en inglés), relativa al plomo $\left(1.5 \mu \mathrm{g} / \mathrm{m}^{3}\right.$ sobre un promedio trimestral). Los controles reguladores de las partículas de materia también han ayudado a reducir las emisiones de dicho elemento a partir de las fuentes estacionarias. Además, la EPA ha aplicado estándares con respecto a la tecnología de control máximo que se puede lograr (MACT) a las fundidoras de plomo secundarias, de acuerdo con las enmiendas que se hicieran en 1990 a la Ley en Favor del Aire Limpio (Clear Air Act). ${ }^{16} \mathrm{La}$



Fuente: Referencia 1

Figura 2. Gasolina con plomo vs niveles de plomo en el aire en los Estados Unidos de América

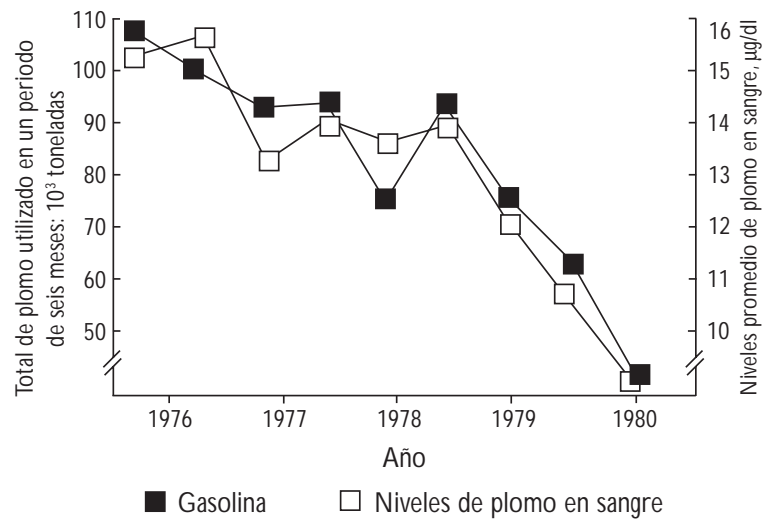

Fuente: Referencia 11

Figura 3. Gasolina con plomo vs niveles de plomo en SANGre en los Estados Unidos de América

combinación de estas medidas ha resultado muy eficaz en la reducción de la emisión de plomo a partir de fuentes estacionarias. Asimismo, la cantidad de grandes fuentes puntuales que podrían verse afectadas por un NAAQS enmendado es pequeña, y está geográficamente limitada. ${ }^{2}$ Así entonces, y partiendo de la suposición de que las normas existentes sean reforzadas, sería muy poco el provecho que se obtendría si se hacen más estrictas las NAAQS vinculadas con el metal. En vez de esto, se han concentrado los esfuerzos para disminuir las emisiones de plomo a partir del número reducido de las fuentes puntuales restantes, identificadas ya como causantes de problemas de emisión local en los EUA.

\section{El plomo en el agua potable}

En los EUA, al igual que en muchos otros sitios, el problema del contenido de este metal en el agua potable se debe, básicamente, a la contaminación por componentes del sistema de distribución, o de la tubería con plomo, y no a la fuente de agua en sí misma. Dicho elemento se ha utilizado en las líneas centrales de servicio, en los conectores, las tuberías para las residencias, la soldadura, la amalgama en el metal de las instalaciones fijas y de los accesorios e, inclusive, en algunos componentes de plástico. Cuando elementos como la acidez, la alcalinidad, la temperatura, la antigüedad de los materiales, y otros más, dan lugar a una reacción química corrosiva con estos materiales, el plomo puede gotear en el agua en varios puntos localiza- 
dos entre la fuente (por ejemplo, un distribuidor de agua potable público) y el destino final (por ejemplo, la llave de agua de la cocina). Estos factores contribuyen a que exista una considerable variabilidad en los niveles de plomo entre los sistemas de distribución de agua e inclusive entre las distintas casas que dependen de un mismo sistema. Tomando en cuenta estas fuentes de variabilidad, la USEPA estableció una Norma Nacional Básica para el Agua Potable (NPDWR, por sus siglas en inglés) en cuanto al contenido de plomo, y que establece la obligación de monitorear su presencia en las llaves de agua de las casas identificadas como de alto riesgo. ${ }^{17}$ De manera más específica, se establece que habrán de colectarse muestras del primer chorro de agua que sale, en aquellos sitios con probabilidad de estar altamente contaminados por plomo, debido a factores conocidos como su presencia en las líneas centrales de servicio, o bien a la soldadura. El agua del primer chorro es aquella que se extrae de la llave una vez transcurridas por lo menos seis horas de no haberla abierto. Partiendo de un muestreo dependiente del tamaño de la población, deberán emprenderse varias acciones para reducir la corrosividad del agua en los sistemas públicos de su distribución que contengan niveles de plomo superiores a los 15 $\mu \mathrm{g} / \mathrm{dl}$ en más de $10 \%$ de las muestras. Los abastecedores de este elemento también pueden llegar a tener la obligación de distribuir material educativo cuya información para los consumidores pretenda reducir su potencial exposición al plomo. Además, dependiendo de la gravedad del problema, a los abastecedores del servicio de agua se les puede solicitar que remplacen las conexiones que contienen plomo, en caso de no obtener buenos resultados con el tratamiento para controlar la corrosión.

En 1986, ${ }^{18}$ antes que la EPA promulgara la Norma Nacional Básica para el Agua Potable, en relación con el plomo -emitida en 1991-, el Congreso de los EUA prohibió la instalación de tuberías con contenidos del metal, así como que la soldadura de plomo estuviese en contacto con el agua potable; en $1988,{ }^{19}$ el Congreso hizo un recordatorio y prohibió el uso de enfriadores de agua que tuviesen plomo en sus componentes. En fechas más recientes, las enmiendas de 1996 a la Ley para el Consumo Seguro de Agua Potable (SDWA, por sus siglas en inglés), especificaron que "ninguna persona puede utilizar un tubo, un tubo o instalación de plomería o accesorio, soldadura, o bien cualquier fundente, en la instalación o reparación de un sistema público de distribución de agua o bien en algún trabajo de plomería en una instalación residencial o no residencial que suministre agua para consumo humano, que contenga plomo" ${ }^{20}$ Inclusive en las enmiendas de 1996 se declaró ilegal la venta de cualquier tipo de tubería, accesorio o instalación "que no estuviese libre de cualquier contenido de plomo", a partir del 6 de agosto de 1998. Para mayor información sobre éstas y otras medidas se puede consultar el siguiente sitio en la red: http:/ /www.epa.gov/safewater/standard/ plumbing.html

\section{Experiencias que pueden aprovecharse}

Uno de los principios que destacan de la anterior discusión es que resulta mucho más fácil desarrollar políticas y normas efectivas cuando se tiene una comprensión clara y coherente de los riesgos y peligros que implica la presencia de este metal, sobre todo en niveles de exposición relativamente bajos. Podemos afirmar que prácticamente no existe nadie que ponga en duda su toxicidad. Sin embargo, cuando se trata de establecer en qué nivel la exposición comienza a significar un peligro para la salud, surgen acalorados debates. El hecho de ilustrar de manera gráfica, así como en términos financieros, las implicaciones que tiene la exposición de bajo nivel a plomo, puede ayudar a los encargados de elaborar políticas a comprender mejor la idea de que tomar medidas para reducir la exposición a éste puede aportar beneficios que exceden los costos que dicha reducción implica. Asimismo, es importante poner a disposición del público en general información científica precisa acerca de este tema. Un público informado puede servir no sólo como incentivo para que el gobierno concrete acciones; también puede tomar medidas, desde el punto de vista individual, para reducir su propia exposición a plomo, así como los riesgos potenciales a la salud.

La experiencia obtenida en los EUA con respecto al plomo en el aire pone de manifiesto que, en ocasiones, se logra mejorar la calidad del aire como efecto secundario de las normas impuestas para solucionar otros problemas. En este caso, el beneficio secundario resultó de la introducción de gasolina sin plomo para consumo en los vehículos equipados con un catalizador. Otro beneficio secundario consistió en el establecimiento de controles para las partículas de materia, que no estaban dirigidos específicamente a las emisiones de partículas de plomo. Lo que cabe resaltar aquí es que se pueden obtener múltiples beneficios a partir de una norma dada. Ciertamente, a pesar de que estos efectos secundarios pueden considerarse benéficos, debe reconocerse la posibilidad de que ocurra exactamente lo contrario; por ejemplo, que se presenten efectos indeseados como consecuencia del establecimiento 
de una medida que parecía indicada. Así, si se elimina el plomo de la gasolina, probablemente será necesario aumentar el contenido de otras sustancias o bien sustituirlas para lograr el octanaje necesario y que el motor tenga el desempeño deseado. Entre las posibilidades para incrementar el porcentaje de octanaje están los aromáticos (por ejemplo, el benceno), los éteres (por ejemplo, MTBE), los alcoholes (por ejemplo, el metanol y el etanol) y otros metales (por ejemplo, MMT) y alquilatos (por ejemplo, iso-octano). Cada una de estas alternativas al uso de este elemento presenta ciertas características que es necesario conocer a fondo, de tal manera que la solución de un problema (el plomo) no implique la aparición de otro en su lugar. Lo anterior no significa que cualquiera de estas alternativas a su uso suponga necesariamente un problema de salud pública más grave que los que ya ocasiona pero, puesto que uno $\mathrm{u}$ otro aditivo al combustible implica problemas potenciales, deberán tomarse medidas para mitigar estos últimos.

Al comparar las normas sobre el contenido del metal en el aire y en el agua se observa una diferencia en las medidas de control: las hay de carácter centralizado y, otras, descentralizado. Así, por ejemplo, la eliminación paulatina de la gasolina con plomo, en todo el país, constituye una medida centralizada. Este tipo de acción reduce básicamente la cantidad del elemento disponible que habrá de dispersarse en el entorno. Efectivamente, "ataja" el plomo en la fuente misma, a diferencia de lo que sucede cuando se intenta manejar el problema una vez que éste ya se ha introducido al ambiente. Como ejemplo de esta última situación está la presencia de sus componentes en las instalaciones y sistemas de plomería que distribuyen el agua potable. Puede resultar bastante difícil y costoso cambiar todos esos componentes, o bien retirarlos de la venta. Empero, en ciertos casos, como por ejemplo, los enfriadores de agua recubiertos con plomo, ya se ha dado inicio a las acciones para retirarlos del uso. Para poder tratar con el problema, mucho más amplio, que representan los componentes del metal en los sistemas de plomería residenciales, la EPA recurrió a un enfoque centralizado en la medida en que los abastecedores de agua estaban implicados, aunque también buscó aplicar medidas correctivas de manera descentralizada. El ataque centralizado del problema era que a los abastecedores de agua se les podía pedir que redujeran la corrosividad de la que distribuían entre sus clientes. Sin embargo, en caso de que este enfoque no bastara, también se les podía pedir que cambiaran las tuberías de abastecimiento y conexiones con plomo, en el punto de distribución a las residencias. La idea de poner información de carácter educativo al alcance de la población puede ser una táctica centralizada; sin embargo, el uso que hace cada individuo de dicha información es, en última instancia, una acción descentralizada. Los métodos centralizados, o bien los descentralizados, pueden ser efectivos, en mayor o menor medida, dependiendo de la naturaleza de la fuente de exposición a plomo, así como de los recursos disponibles para manejarla.

Por último, en este documento se ha enfocado la atención a medios como el agua y el aire, pero debe reconocerse que la exposición a plomo puede deberse a muchas otras fuentes también, y que ésta es acumulativa, tanto en las vías como con el transcurso del tiempo. Así entonces, será necesario identificar las fuentes más importantes de exposición, ya sea individual, local, regional o bien nacionalmente. Por otra parte, las contribuciones relativas de las distintas fuentes pueden variar, sobre todo cuando resultan efectivas las medidas para controlar alguna de éstas, de tal manera que las otras fuentes ocupen, proporcionalmente, un lugar más grande en la exposición total.

El uso intensivo del plomo durante siglos ha dejado un legado nocivo; para resolver esta situación se requiere de una respuesta de salud pública concertada. No obstante, con dedicación y análisis inteligentes, se puede cumplir con este reto.

\section{Agradecimientos}

Los puntos de vista vertidos en este documento son los de los autores y no reflejan necesariamente las opiniones o políticas de la Agencia Norteamericana para la Protección del Ambiente. Agradecemos los útiles comentarios de los encargados de revisar este trabajo.

\section{Referencias}

1. Davis JM, Elias RW, Grant LD. Efforts to reduce lead exposure in the United States. En:Yasui M, Strong MJ, 0 ta K, Verity MA, Ed. Mineral and metal neurotoxicology. Boca Raton (FL): CRC Press, 1997:285-293.

2. D avis JM, Elias RW, Grant LD. Current issues in human lead exposure and regulation of lead. N eurotoxicology 1993;14(2-3):15-27.

3. US Environmental Protection A gency. Air quality criteria for lead. Research Triangle Park (N C): 0 ffice of Health and Environmental Assessment, Environmental C riteria and Assessment 0 ffice 1986; EPA report no. EPA-600/8-83/028aF-dF. 4v. Available from: N TIS, Springfield, VA; PB87-142378.

4. US Environmental Protection A gency. Air quality criteria for lead: Supplement to the 1986 addendum. Research Triangle Park (N C): 0 ffice of Health and Environmental Assessment, Environmental Criteria and Assessment 0 ffice1990; EPA Report no. EPA/600/8-89/049F,. Available from: N TIS, Springfield,VA; PB91-138420/X AB. 
5. US C enters for Disease Control. Preventing lead poisoning in young children: A statement by the Centers for Disease Control - 0 ctober 1991.A tlanta (GA): US D epartment of H ealth \& Human Services, Public Health Service, 1991.

6.A gency for Toxic Substances and Disease Registry. The nature and extent of lead poisoning in children in the U nited States:A report to Congress. Atlanta (GA): US D epartment of Health and Human Services, Public Health Service, 1988. Available from: N TIS, Springfield,VA; PB89$100184 / \mathrm{XAB}$.

7.A gency for Toxic Substances and D isease Registry.Toxicological profile for lead. Atlanta (GA): US D epartment of Health and Human Services, Public Health Service, 1999. Available on-line at: http:// www.atsdr.cdc.gov/toxprofiles/tp13.html.

8.W orld Health $O$ rganization. Inorganic lead. G inebra:W orld Health O rganization, International Programme on Chemical Safety 1995; Environmental health criteria 165.

9. D avis JM , Elias RW. Risk assessment of metals. En: C hang LW, Magos L, Suzuki T, Ed. Toxicology of metals. Boca Raton (FL): CRC Lewis Publishers, 1996:55-67.

10. Schwartz J. Societal benefits of reducing lead exposure. En: George AM, Ed. Lead poisoning prevention \& treatment: Implementing a national program in developing countries. Bangalore, India:The George Foundation, 1999:47-64.

11. Annest JL.Trends in the blood lead levels of the US population:The Second $N$ ational Health and N utrition Examination Survey (N HAN ES1I) 1976-1980. En: Rutter M, Russell-Jones R, Ed. Lead versus health: Sources and effects of low level lead exposure. N ueva York (N Y): John W iley and Sons, 1983:33-58.
12.W ietlisbach V, Rickenbach M, Berode M, Guillemin M.Time trend and determinants of blood lead levels in a Swiss population over a transition period (1984-1993) from leaded to unleaded gasoline use. Environ Res 1995;68:82-90.

13. D avis JM, Svendsgaard DJ. Lead and child development. $N$ ature 1987;329:297-300.

14. Federal Register. Regulation of fuel and fuel additives; gasoline lead content. F. R. (March 7, 1985)50:9386-9408.

15. US Environmental Protection A gency. Clean Air Act, as amended by PL 101-549, N ovember 15, 1990. U. S. C ode 42: sect. 7401-7671q. 16. US Environmental Protection Agency. $N$ ational air quality and emissions trends report, 1995. Research Triangle Park (NC): 0 ffice of Air Q uality Planning and Standards, Emissions Monitoring and Analysis Division 1996; Report no. EPA/454/R-96-005. Available from: NTIS, Springfield,VA; PB97-127500/X AB.

17. Federal Register. $N$ ational emission standards for hazardous air pollutants from secondary lead smelting. F. R. (June 23, 1995)60:32, 587-532,601.

18. Federal Register. Maximum contaminant level goals and national primary drinking water regulations for lead and copper: Final rule. F. R. (June 7, 1991)56:26460-26564.

19. US Environmental Protection A gency. Safe D rinking W ater Act, as amended by PL 99-339, June 19, 1986. U. S. Code 42: sect. 300 fe seq. 20. US Environmental Protection A gency. Lead Contamination C ontrol Act, PL 100-572, 0 ctober 31, 1988. U. S. Code 42: sect. 300j-21-300j-26. 21. US Environmental Protection A gency. Safe D rinking W ater Act, as amended by PL 104-182, August 6, 1996. U. S. Code 42: sect. 1417(a). 